\title{
Evolutionary Hysteresis and Ratchets in the Evolution of Periodical Cicadas
}

\section{Toivonen, Jaakko}

2019-07-01

Toivonen , J \& Fromhage , L 2019 , ' Evolutionary Hysteresis and Ratchets in the Evolution of Periodical Cicadas ' , American Naturalist , vol. 194 , no. 1 , pp. 38-46 . https://doi.org/10.1086/703563

http://hdl.handle.net/10138/304514

https://doi.org/10.1086/703563

cc_by_nc

publishedVersion

Downloaded from Helda, University of Helsinki institutional repository.

This is an electronic reprint of the original article.

This reprint may differ from the original in pagination and typographic detail.

Please cite the original version. 


\title{
Evolutionary Hysteresis and Ratchets in the Evolution of Periodical Cicadas
}

\author{
Jaakko Toivonen ${ }^{1,2, \star}$ and Lutz Fromhage ${ }^{2}$ \\ 1. Department of Computer Science, University of Helsinki, FI-00014 University of Helsinki, Finland; 2. Department of Biological and \\ Environmental Science, University of Jyväskylä, FI-40014 University of Jyväskylä, Finland
}

Submitted November 6, 2018; Accepted February 12, 2019; Electronically published May 20, 2019

Online enhancements: appendix, Matlab code.

\begin{abstract}
A BSTRACT: It has been previously hypothesized that the perfectly synchronized mass emergence of periodical cicadas (Magicicada spp.) evolved as a result of a switch from size-based to age-based emergence. In the former case, cicada nymphs emerge immediately (at the first opportunity) on reaching maturity, whereas in the latter case, nymphs wait in order to emerge at a specific age. Here we use an individualbased model to simulate the cicada life cycle and to study the evolution of periodicity. We find that if age-based emergence evolves in a constant abiotic environment, it typically results in a population that is protoperiodic, and synchronous emergence of the whole population is not achieved. However, perfect periodicity and synchronous emergence can be attained, if the abiotic environment changes back and forth between favorable and unfavorable conditions (hysteresis). Furthermore, once age-based emergence evolves, generally it can only be invaded by other age-based emergence strategies with longer cycle lengths (evolutionary ratchet). Together, these mechanisms promote the evolution of long periodic life cycles and synchronous emergence in the Magicicada. We discuss how our results connect to previous theories and recent phylogenetic studies on Magicicada evolution.
\end{abstract}

Keywords: periodicity, synchronous life cycle, individual-based simulation, Magicicada, temporally varying environments.

\section{Introduction}

Periodical cicadas (Magicicada spp.) are known for their periodical life cycles and tremendous synchronized mass emergence once every 13 or 17 years, with the precise cycle length depending on the species. Periodical cicada nymphs live underground until the final year of their life cycle, when all nymphs emerge from the ground. The massive synchronous emergence of periodical cicadas reduces the per capita death rate as they satiate predators (Karban 1982). After

\footnotetext{
* Corresponding author; email: jaakko.toivonen@alumni.helsinki.fi. ORCIDs: Toivonen, https://orcid.org/0000-0002-5444-8877; Fromhage, https://orcid.org/0000-0001-5560-6673.
}

Am. Nat. 2019. Vol. 194, pp. 38-46. (C) 2019 by The University of Chicago. 0003-0147/2019/19401-58854\$15.00. All rights reserved.

DOI: $10.1086 / 703563$ molting, adult cicadas proceed to mate and reproduce, and after laying eggs they perish. Once nymphs hatch from the eggs, they burrow into the ground, thus beginning a new population cycle. The ecology of the periodical cicadas and how the long, synchronous, and prime-numbered life cycles evolved has been the subject of many studies (e.g., Alexander and Moore 1962; Dybas and Davis 1962; Dybas and Lloyd 1962, 1974; Lloyd and Dybas 1966a, 1966b; White and Lloyd 1975; White et al. 1979; Karban 1984; Williams and Simon 1995; Marshall and Cooley 2000; Sota et al. 2013). In this article, we demonstrate how two interesting evolutionary mechanisms that perhaps have not received enough attention so far-namely, hysteresis (Kisdi and Geritz 1999; Ronce and Kirkpatrick 2001; Fortelius et al. 2015; Osmond and Klausmeier 2017) and ratchet mechanisms (Levinton 2001; Wahl 2002; Valkenburgh 2007; Ritterskamp et al. 2016) — could both have contributed to the evolution of periodicity and long life cycles in the Magicicada.

Periodicity in insects is rare, but other examples besides the Magicicada do exist, such as oak eggar moths, May beetles, pine bark bugs, arctic chironomids, woolly bear moths, the mayfly Habrophlebia vibrans, the midge Chironomus anthracinus, and pine-resin gall moths Retinia resinella (see Martin and Simon 1990; Heliövaara et al. 1994 and references therein). On the other hand, protoperiodicity is common in insects (Heliövaara et al. 1994). This means that emergence densities vary such that some years have many individuals emerging and some years have only a few individuals emerging.

The genetic basis of the timing of emergence in periodical cicadas remains unknown (Williams and Simon 1995). Field studies of the Magicicada have shown that individuals grow at significantly differing rates (Lloyd and Dybas 1966b; White and Lloyd 1975), yet they all emerge simultaneously. Thus their emergence appears to be determined by a fixed time interval, and fast-growing individuals likely delay emergence to synchronize with the others. In contrast, annual cicadas have varying life-cycle lengths. This could be due to 
individuals growing at different rates and emerging immediately on reaching maturity. Alternatively, it could represent a bet-hedging strategy, where emergence is genetically determined at a specific age, but this age (randomly) varies between individuals, so that emergence is spread out over multiple years to reduce the risk of emerging in a particularly bad year (Martin and Simon 1990).

It has been suggested that the periodical cicadas evolved from annual cicadas (Martin and Simon 1990) by switching from size-based to age-based emergence (Yoshimura 1997; Ito et al. 2015). In the former case, individuals emerge immediately on reaching some threshold body size, while in the latter case, individuals wait in the ground until they reach some threshold age. Previously, Ito et al. (2015) and Toivonen and Fromhage (2018) used similar individualbased models (IBM) to investigate under what conditions an age-based emergence trait could invade an ancestral (resident) population that follows the size-based emergence rule. They explicitly modeled body-size growth and assumed that fecundity is an increasing function of body size (this assumption was implicit in Ito et al. 2015). They assumed that age-based individuals were always able to emerge perfectly on schedule and that only their fecundity was affected by chance variability in individual yearly growth rates. In this setting, there is a trade-off between fecundity and survival. Toivonen and Fromhage (2018) found that, typically, the threshold body size for emergence evolves such that it balances this trade-off. Further, they found that age-based emergence is selected for only in situations where predatorinduced mortality is high relative to the cicada population size and that this causes the resident population to become protoperiodical. If the interval of time between peak emergence years is somewhat consistent in length, then age-based emergence may be favored as it allows fast-growing individuals to avoid emergence on low-emergence-density years (when predator-induced mortality is high). Ito et al. (2015) did not consider the potential evolution of the threshold body size, which may have confounded their results because it is not clear whether age-based emergence strategies in their model benefit from synchronous emergence or whether they are favored simply because they find a better balance regarding the fecundity-survival trade-off. Furthermore, the threshold body size should be under natural selection, and given that it is assumed to be the ancestral trait, it should have had time to evolve to balance the trade-off. Therefore, picking an arbitrary size threshold is likely to be unrealistic.

In contrast to the articles by Ito et al. (2015) and Toivonen and Fromhage (2018), here we model growth as a process toward reproductive maturity. We assume that even agebased individuals who have already reached their target age cannot emerge before also reaching the requisite body size for reproductive maturity; hence there is no trade-off between fecundity and survival and, further, no ambiguity about selec- tion between different threshold values. In the model presented here, age-based individuals may not be able to automatically emerge in perfect synchrony, as some individuals may grow too slowly and not reach maturity in time. Ultimately, it is difficult to say which model for growth and emergence better captures the growth process and emergence trigger of the Magicicada, as very little is known of the genetic basis of emergence for them. However, there have been observations of delayed emergence in nutrient-poor environments (White and Lloyd 1975, 1979), where it is likely that growth would have been slow. Such observations may lend support for our approach to modeling the growth process and emergence triggers in the Magicicada.

Since in the present model age-based emergence does not automatically lead to perfect synchrony of emergence, the question arises as to how it is possible that the Magicicada do exhibit near-perfect synchrony. A possible solution could be through history dependence, that is, hysteresis: age-based emergence may first evolve in a slow-growth environment and then environmental change toward fast-growth environments could allow the population to become fully synchronous as all individuals are able to reach maturity in time to emerge on schedule. This idea is particularly interesting as it has been previously hypothesized that periodical cicadas originally evolved during the Pleistocene ice age, when environmental conditions were harsh, cicada nymph growth rates were slow, and cicada life-cycles were elongated (Yoshimura 1997). To test this hypothesis, we investigate different scenarios of slow temporal variation in environmental conditions to determine when age-based emergence can evolve and develop perfect synchrony.

\section{Methods}

For this study, we use the same individual-based model that was previously used by Toivonen and Fromhage (2018) but with one crucial change. Toivonen and Fromhage (2018) explicitly modeled body-size growth while assuming that (age-based) emergence is possible at any size. Here we model growth as a process toward reproductive maturity, which is a requirement for emergence that is reached at a given target size. We assume that individuals accrue maturity by random yearly increments, and once the total sum of growth exceeds some threshold value, we deem the individual as mature. Since an individual cannot reproduce before reaching maturity, we assume that age-based individuals do not emerge from the ground until they have both reached some threshold age and reproductive maturity. In contrast, size-based individuals emerge at the first opportunity once they have reached maturity. While seemingly a small change, the effect is significant in that now age-based individuals of the same cohort do not automatically emerge synchronously as they may not 
always be able to emerge precisely according to the cycle length defined by their trait.

Here we give a brief layout of the individual-based model; a more detailed description is given by Toivonen and Fromhage (2018). Each iteration of the main simulation loop corresponds to 1 year in the cicada life cycle. We begin each simulation by creating an initial population of female nymphs, with each individual having personal values for certain characteristics (genotype, phenotype, body size, and age). We assume a one-locus, two-alleles genetic system. Each individual in the initial population is assumed to have two copies of the allele coding for size-based emergence.

For each round of the main simulation loop, we first update the value of an environmental parameter. This allows us to model temporally changing environments. Then the population of nymph individuals undergoes density-dependent competition: periodical cicada nymphs are likely regulated by the available resources and space (White et al. 1979; Karban 1984). We calculate a density-dependent probability of survival and perform a biased coin toss for each individual. Each individual determined to have died is removed from the population.

Next we update the age and body size of each surviving nymph. The age of each individual is increased by one, and the body size is increased with a random positive increment drawn from the lognormal distribution $X=\exp \left(\mathcal{N}\left(\mu, \sigma^{2}\right)\right)$, where $\mu$ is the mean and $\sigma$ the standard deviation of the normal distribution. We assume that $\sigma$ is a constant and $\mu$ may vary over time depending on the environmental scenario. This reflects the assumption that environmental conditions affect growth rates: the median growth rate is $e^{\mu}$, the mean growth rate is $e^{\mu+\sigma^{2} / 2}$, and the variance is $e^{2 \mu+\sigma^{2}}\left(e^{\sigma^{2}}-1\right)$ (Smith and Merceret 2000). The lognormal distribution is common in natural growth processes (Koch 1966; Grönholm and Annila 2007). Mechanistically, it can be viewed as modeling a situation where yearly growth is concentrated to a growth season (e.g., spring) during which growth is exponential with growth rate $\mu$. However, we do not believe that our results are dependent on the particular choice of a growth distribution and that other growth distributions could be used just as well as long as growth rates are positive and do not have an unreasonably large variance. After increasing age and body size, we check for each nymph whether it has reached its emergence condition. Each such individual is removed from the nymph population and added to the adult population of the current year.

The following step is adult emergence. Emerging cicadas suffer density-dependent mortality due to predation so that probability of survival is higher in years of high emergence density (Karban 1982). We calculate a density-dependent survival probability and then flip a biased coin for each individual to determine whether it survives predation or not. We model predation with a Holling type II functional re- sponse, which models predator satiation (Holling 1959). The surviving adults each produce a fixed number of offspring. We assume sexual mating with male genotype ratios identical to female ratios. We assume a fixed mutation probability that may change a given allele of an offspring so that a size-based emergence allele becomes an age-based allele with a cycle length that is a random integer between 1 and 25. Mutation to an age-based allele may either change it to a size-based allele or it may change the cycle length by 1 year to a shorter or a longer cycle. We assume that size-based emergence is a dominant trait. Also, if an age-based individual has two alleles that code for different cycle lengths, we assume that the shorter cycle length dominates. After reproduction, new offspring are added to the nymph population and all adults die.

The Matlab codes for the IBM and the parameter values used for all of our simulations are available online. ${ }^{1}$

\section{Results}

In order to demonstrate the hysteresis and ratchet effects, we study three different types of scenarios for temporally changing abiotic environments: constant, unimodal, and bimodal environments. We use the lognormal mean nymph growth rate $\mu$ as a proxy for the abiotic environment. Environments in which average growth is relatively fast (high $\mu$ ) are referred to as favorable environments, and environments in which average growth is relatively slow (low $\mu$ ) are referred to as unfavorable environments. We run simulations to determine how different environmental scenarios affect the invasion of age-based emergence traits and the evolution of synchronous emergence.

\section{Constant Environment}

Here we assume that the abiotic environment remains constant throughout a simulation. We run 10 independent simulations for a range of values of $\mu$. The results are summarized in figure 1.

We observe that in favorable environments $(\mu \geq-0.7)$, age-based emergence traits do not invade. However, in unfavorable environments $(\mu<-0.8)$, they do, with the invading cycle length tending to be longer toward conditions where growth is slow (decreasing values of $\mu$ ). This shows that when age-based emergence evolves, there is strong selection for a particular cycle length that depends on the environment.

As the environment becomes more unfavorable ( $\mu$ decreases), the average time it takes to reach maturity increases due to slower growth, and thus average life-cycle length in-

1. Code that appears in The American Naturalist is provided as a convenience to readers. It has not necessarily been tested as part of peer review. 

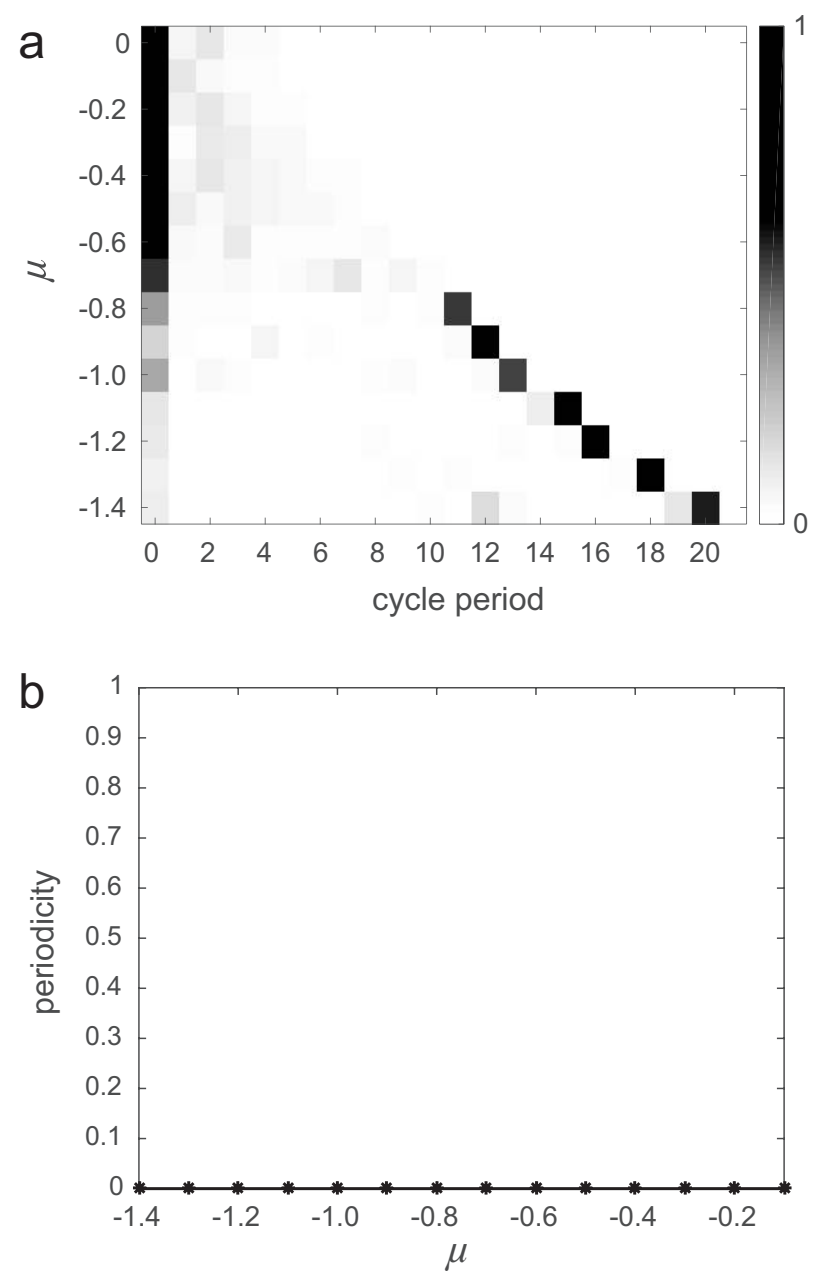

Figure 1: Results from the constant environmental scenario. $a$, Average proportion of different emergence strategies at the end of simulation runs for a range of values of the environmental proxy variable $\mu$. The range $\mu \in[-1.4,0]$ corresponds to a range of median yearly growth rates between $e^{-1.4} \approx 0.2466$ and 1 , with the maturity threshold size set at 5 (arbitrary units). The value 0 for the "cycle period" variable refers to a size-based emergence strategy, while the rest of the values indicate age-based emergence strategies with corresponding cycle lengths. For $\mu>-0.8$, the majority of the population follows the size-based emergence trait. For $\mu \leq-0.8$, the majority of the population follows an age-based emergence trait, and the cycle length of the population grows as $\mu$ decreases (indicating slower growth to maturity). $b$, Average proportion of simulation runs that ended with a fully periodic population (with synchronous emergence). In a constant environment, full periodicity is never achieved.

creases. This leads to lower total population and emergence sizes for the resident size-based population (fig. Ala, A1b; figs. A1-A3 are available online) because individuals have to endure competition for an extended period of time before they are able to reproduce. As the size of the emerging adult population becomes smaller, the impact of a constant predator population becomes proportionally bigger. Eventu- ally this causes the resident size-based population to change from nonperiodic to protoperiodic emergence (fig. A1c): the population is not synchronous in emergence but does exhibit periodically high emergence densities between years of no emergence at all (fig. A2). The average time between peak emergence years depends on the environment so that, due to longer maturation times, more unfavorable environments produce longer protoperiodic cycle lengths (fig. A1d).

When yearly emergence densities are constant, there is no reason for a mature individual to delay emergence and risk mortality in the ground for an added time. However, when the population has become protoperiodical, an agebased emergence trait with a suitable cycle length becomes advantageous: the strategy allows fast-maturing individuals not to emerge in a year of low emergence density and instead wait for a better year when the large population of simultaneously emerging conspecifics offers safety in numbers from predation. We observe that in the range $\mu=[-0.7,-0.5]$, the resident population becomes protoperiodic (fig. A1c), but age-based emergence does not successfully invade (fig. 1a). Further tests with longer simulation times indicate that agebased emergence will evolve in this range as well, but the transient phase lasts for a longer time than in the range $\mu \leq-0.8$.

Up to this point, the results are similar to those of Toivonen and Fromhage (2018). However, contrary to this previous work, here we find that when an age-based emergence trait invades in unfavorable environments, the resulting population does not become completely synchronous, but rather it remains protoperiodical. This happens because a significant proportion of the age-based population is not able to reach maturity in time to emerge immediately at their genetically determined target age. Then emergence is spread out over some interval of consecutive years. This is in contrast to the article by Toivonen and Fromhage (2018), in which a population following the age-based emergence rule would always exhibit complete synchrony of emergence.

\section{Unimodal Environment}

Here we assume an environmental scenario in which the environment at first is favorable $(\mu=0)$ and then becomes progressively more unfavorable, reaching a plateau at $\mu=\mu_{1}$. From there the environment stays constant for a period of time and then begins to improve again, ultimately changing back to the original favorable environment (fig. A3a). We run 10 independent simulations for a range of values of $\mu_{1}$. The results are summarized in figure 2.

Similar to the case with a constant environment, we again find that age-based emergence can evolve if the environment becomes relatively unfavorable. However, if age-based emergence evolves, it is maintained even when the environment becomes favorable again. This occurs because the improving environmental conditions allow individuals to con- 

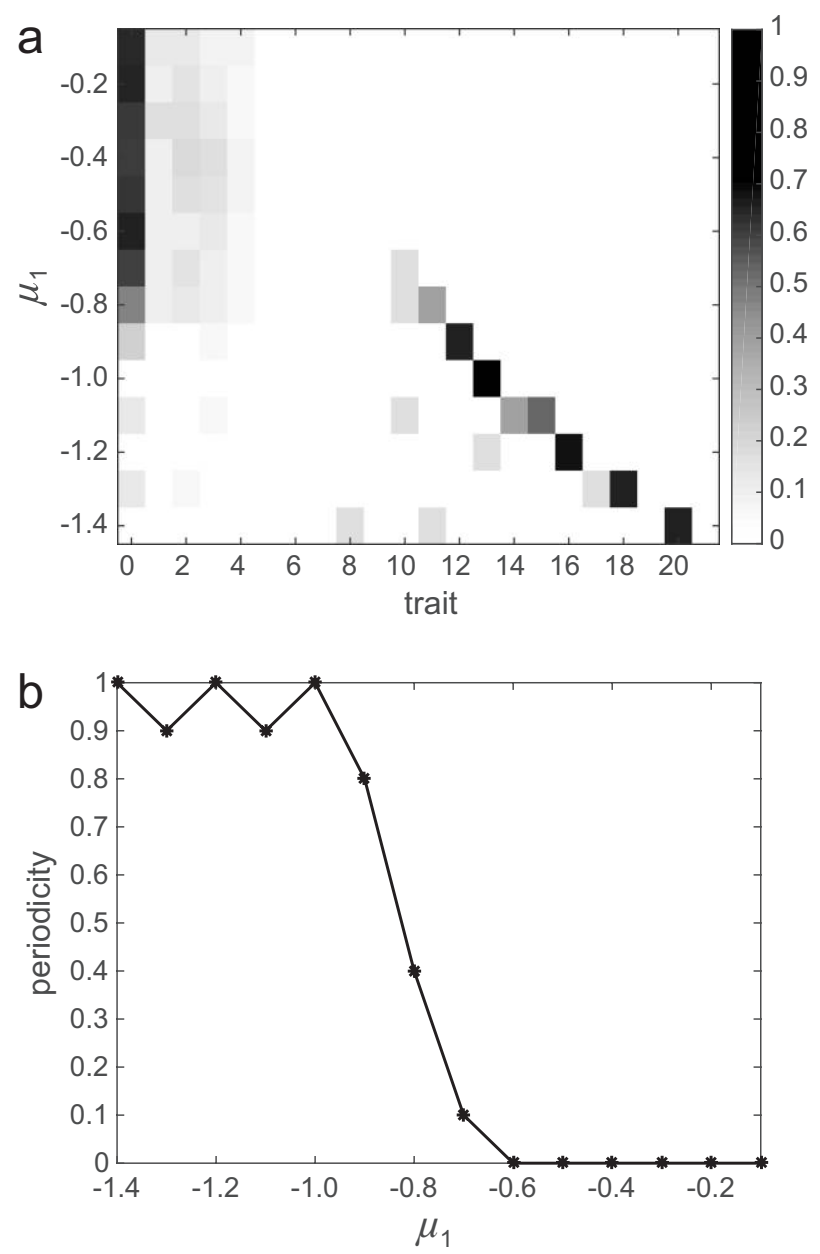

Figure 2: Results from the unimodal environmental scenario. $a$, Average proportion of different emergence strategies at the end of simulation runs for a range of values of the environmental plateau $\mu_{1}$. The range $\mu_{1} \in[-1.4,-0.1]$ corresponds to a range of median yearly growth rates between $e^{-1.4} \approx 0.2466$ and $e^{-0.1} \approx 0.9048$, with the maturity threshold size set at 5 (arbitrary units). The value 0 for the "cycle period" variable refers to a size-based emergence strategy, while the rest of the values indicate age-based emergence strategies with corresponding cycle lengths. For $\mu_{1}>-0.8$, the majority of the population follows the size-based emergence trait. For $\mu_{1}<-0.8$, the majority of the population follows an age-based emergence trait, and the cycle length of the population grows as $\mu_{1}$ decreases (indicating slower growth to maturity). $b$, Average proportion of simulation runs that ended with a fully periodic population (with synchronous emergence). Contrary to the constant environment, in a unimodal environment, it is possible to achieve full periodicity when the population evolves age-based emergence.

sistently reach maturity before or at their age threshold for emergence. Then the population becomes periodic with synchronous emergence. Once this happens, no other cycle length or size-based emergence can evolve, because per capita mortality due to predation is much higher when emerging on a different year than the main cohort. As the age-based traits originate in unfavorable environments, when nymph growth is slow, the cycle length of the age-based trait that evolves is typically much longer than the average time needed to reach maturity in a favorable environment.

Even though we do not observe age-based emergence evolving in constant favorable environments, our results show it can become established in those environments as well under the right circumstances. This type of dependence of the state of the system, not only on the current environment but also on the history of environmental conditions, is known as hysteresis.

\section{Bimodal Environment}

Here we assume that the environment follows a similar pattern as in the unimodal case but now with two valleys of unfavorable environments (fig. A3b). We assume that there are two intervals of constant unfavorable environments, with environmental proxy values $\mu_{1}$ for the first interval and $\mu_{2}$ for the second interval. We run 10 independent simulations for a range of values of $\mu_{2}$ while keeping $\mu_{1}$ fixed. The results are summarized in figure 3 .

We observe that if $\mu_{2} \geq \mu_{1}$-that is, if the second plateau corresponds to a more favorable environment than the first one - then the results are qualitatively similar to the case with a unimodal environment: typically, age-based emergence evolves, the cycle length is predictable based on the value of $\mu_{1}$, and the age-based population at the end of the simulation has developed complete synchrony.

Typically, age-based emergence evolves during the first environmental cycle with a cycle length characteristic to $\mu_{1}$, and perfect synchrony is attained when the environment becomes favorable (as in the unimodal case). We observe that if $\mu_{2}=\mu_{1}$, then the population typically becomes protoperiodic again during the second environmental cycle. This happens because average growth rates have decreased so that a significant proportion of the population is not able to reach maturity in time to emerge according to the age-based trait. However, when the environment begins to improve again, perfect synchrony is restored as individuals begin to mature fast enough so that virtually all individuals are able to reach maturity before attaining the age threshold. Synchronicity of the resident population then prevents the invasion of other age-based traits with shorter cycle lengths (see "Unimodal Environment").

If $\mu_{2}<\mu_{1}$ - that is, if the second plateau corresponds to a more unfavorable environment than the first one - then the population typically develops a longer periodic cycle (fig. 4 shows one example simulation run). Typically, the first cycle of environmental change causes the original size-based population to evolve age-based emergence, and the hysteresis effect allows it to become perfectly synchronous at the end of the cycle. However, during the second cycle of en- 

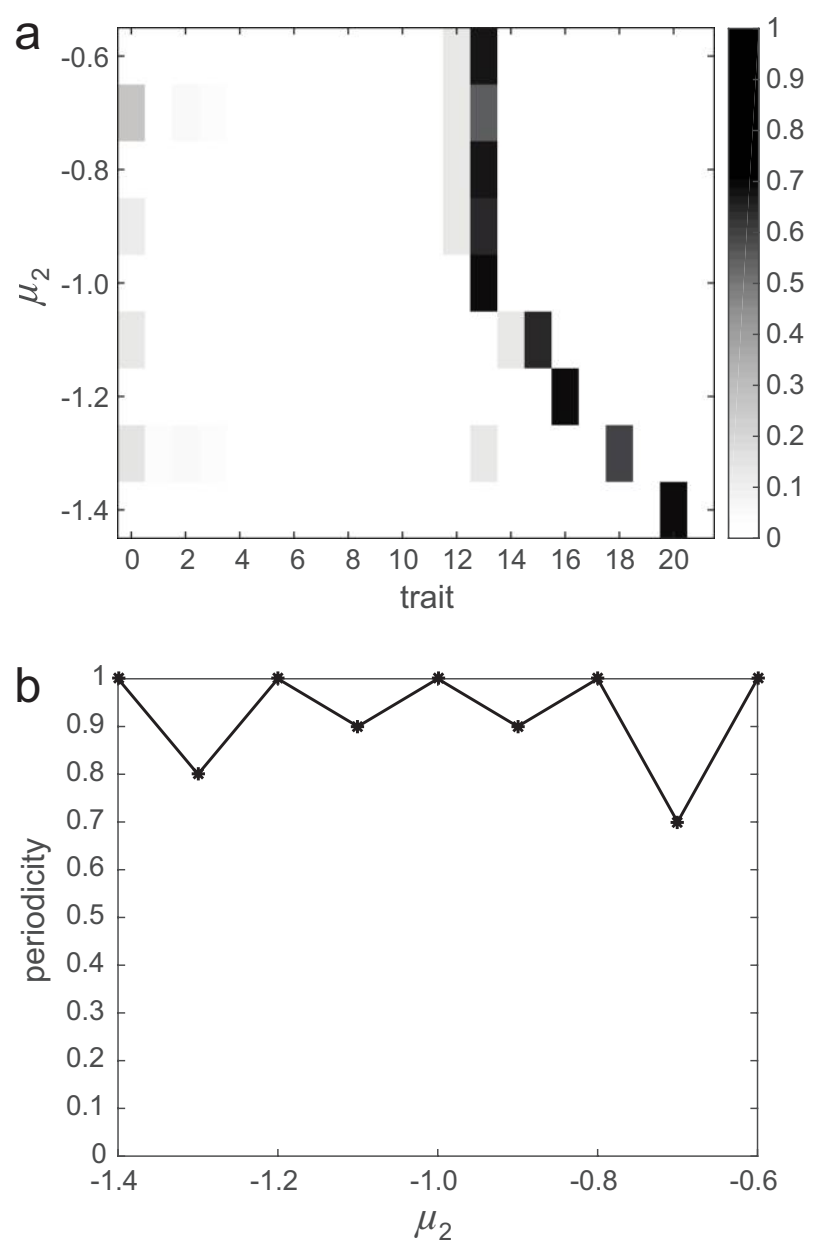

Figure 3: Results from the bimodal environmental scenario. $a$, Average proportion of different emergence strategies at the end of simulation runs for a range of values of the environmental plateau $\mu_{2}$. The range $\mu_{2} \in[-1.4,-0.6]$ corresponds to a range of median yearly growth rates between $e^{-1.4} \approx 0.2466$ and $e^{-0.6} \approx 0.5488$, with the maturity threshold size set at 5 (arbitrary units). The value 0 for the "cycle period" variable refers to a size-based emergence strategy, while the rest of the values indicate age-based emergence strategies with corresponding cycle lengths. The population consistently evolves age-based emergence. When $\mu_{2} \geq \mu_{1}=-1.0$, the cycle length of the population is typically 13 , which is consistent with the constant and unimodal environments (with $\mu=\mu_{1}=-1.0$ ). When $\mu_{2}<\mu_{1}$, the cycle length increases again, as it similarly does in the constant and unimodal environmental scenarios. $b$, Average proportion of simulation runs that ended with a fully periodic population (with synchronous emergence).

vironmental change, once $\mu$ decreases close to $\mu_{1}$, this perfect synchrony breaks down, as a significant proportion of the population does not mature fast enough to emerge on schedule. As $\mu$ keeps decreasing, typically the population evolves size-based emergence again; and once a new plateau in environmental change is reached, that is, $\mu=\mu_{2}$, a new age-based emergence trait with a longer cycle length than previously invades. Once the environmental parameter $\mu$ begins to increase, the population develops perfect synchrony again. We call this the evolutionary ratchet effect: a periodical population can generally evolve only such that the cycle length increases, whereas the opposite change tends not to occur.

While we do observe that size-based emergence can reappear when the synchronous emergence breaks down due to declining environmental conditions, this typically is a transient phase, and age-based emergence evolves again once the environmental condition stabilizes (plateaus) for some interval of time. Further, we note that our model was set up such that size-based emergence is a dominant trait to age-based emergence, and shorter cycle lengths dominate in heterozygotes with two age-based alleles of differing lengths. These choices may hamper the ability of the model population to switch from one age-based trait to a longer cycle, as the latter phenotype is manifested only once both of the two alleles have changed. In contrast, the size-based emergence trait manifests phenotypically immediately once a single mutation has occurred. Other assumptions regarding mutations and dominance might result in a succession of ever-longer age-based traits invading during declining environmental conditions.

\section{Discussion}

In this article, we showcase two generally interesting evolutionary mechanisms that potentially provide novel insights on the evolution of the Magicicada. The first mechanism is hysteresis: perfect periodicity does not evolve in a static environment, but it can evolve if the history of environmental change is suitable. For the present model, age-based emergence only evolves in unfavorable environments (in which nymphal growth is slow) due to low population densities relative to predation pressure. When age-based emergence evolves, we see that the population becomes protoperiodical, but it does not exhibit perfect periodicity because many individuals do not reach maturity in time to emerge synchronously with their cohort. To achieve perfect periodicity, it is necessary for the environment to become more favorable so that nymph growth rates increase. Then it is possible for virtually all individuals to mature in time to emerge synchronously according to the age threshold adopted. Further, competition among nymphs and predation of emerging adults leads to the elimination of all but one cohort (Hoppensteadt and Keller 1976; Bulmer 1977), and thus population emergence becomes fully synchronous.

The second mechanism we showcase is the evolutionary ratchet. We show that once age-based emergence has evolved and the environment is favorable enough so that the population has become fully periodic and synchronous, then typi- 

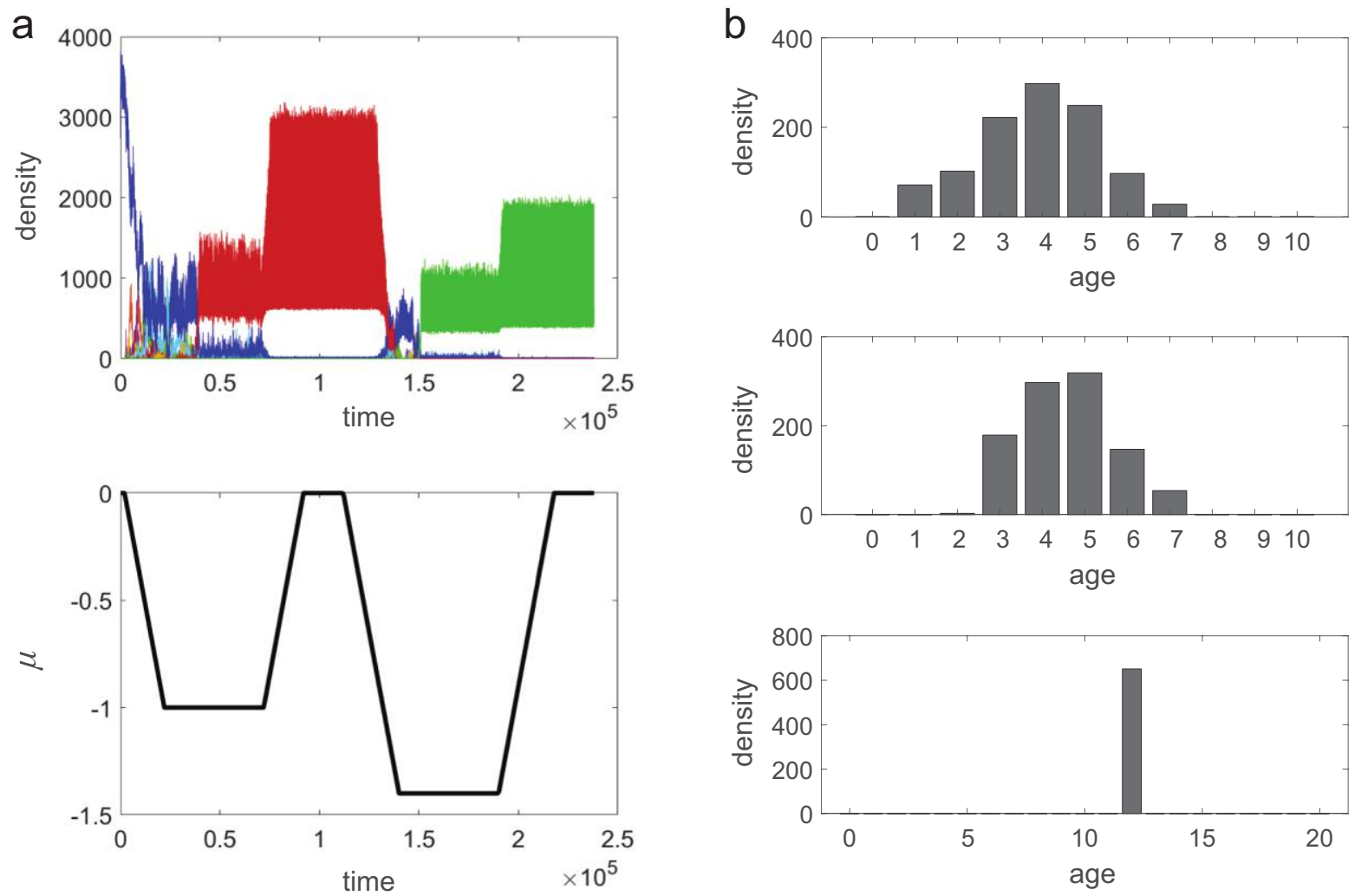

Figure 4: An example simulation run. $a$, Top, population densities of different cycle periods over time. The population density of the sizebased emergence trait is shown in blue. The population densities of age-based traits with a cycle length of 13 and 20 years are shown in red and green, respectively. Both age-based traits are initially protoperiodic but then become fully periodic (synchronous in emergence) once the environmental conditions improve sufficiently. Once the population is periodic, it shows a significant increase in its peak population densities. Bottom, change in the environmental parameter $\mu$ over time. $b$, Age distribution of the population at different times. Top, $t=0.3 \times 10^{5}$. The population mainly consists of individuals with the size-based emergence trait, but it has become protoperiodic: there are no newborns in the population, and the age distribution is not skewed toward younger individuals. Middle, $t=0.5 \times 10^{5}$. Age-based emergence with a cycle length of 13 years has invaded. The population is still protoperiodic, but the age distribution is somewhat less spread out than before. Bottom, $t=10^{5}$. The population has become fully synchronous, with only one age class existing.

cally only longer cycle lengths can evolve, and neither shorter cycle lengths nor size-based emergence can evolve anymore (apart from a possible transient phase between transitions from one cycle length to a longer one; see "Bimodal Environment"). The evolution of a new, longer cycle length requires that the environment again becomes sufficiently unfavorable so that synchronous emergence is broken down (due to many individuals not being able to mature fast enough). Then the population becomes once again protoperiodical, and further environmental change that reduces nymph growth rates can increase the average time between years of peak emergence density. If this occurs, then a new age-based emergence trait with a longer cycle length that is a better match for the current protoperiodical cycle length of the population can invade. If the environment eventually becomes favorable again, then the population returns to synchronous emergence but now with a longer cycle length than originally.
Toivonen and Fromhage (2018) showed that age-based emergence can evolve in any environment, and the determining factor of whether it evolves or not is how large predation pressure is relative to the cicada population density. Our results here agree with Toivonen and Fromhage (2018) in showing that longer life cycles evolve in environments where nymphal growth is relatively slow, and shorter lifecycles evolve when nymphal growth is relatively fast. This also appears consistent with the observation that 17 -year cicadas exist in the northern parts and 13-year cicadas in the southern parts of North America (see, e.g., Cox and Carlton 1988, fig. 1): the availability of nutrition has been shown to affect growth rates in nonperiodical cicadas (Ito and Nagamine 1981), and colder and harsher environments are likely to have less nutrition available. However, in the model of Toivonen and Fromhage (2018), once periodicity evolved and a particular cycle length was attained, it was evolution- 
arily stable and therefore could not change anymore. In contrast, by relaxing that study's assumption that an agebased emergence strategy will be precisely realized under any environmental conditions, we here predict that subsequent changes in cycle length are possible after all. We find that age-based emergence can evolve in a wide range of environmental conditions (most likely, it can evolve in any abiotic environment, but here we did not investigate varying predation pressure), but perfect periodicity evolved only through the hysteresis effect.

It has been suggested that periodical cicadas evolved during the Pleistocene ice age (Cox and Carlton 1988, 2003) by switching from size-based to age-based emergence (Yoshimura 1997; Ito et al. 2015). However, recent phylogenetic studies (Sota et al. 2013) have shown that, while divergence between the 13- and 17-year cycles did happen during the Pleistocene, the original split of the Magicicada genus occurred already circa 3.9 million years ago, which is well before the Pleistocene. Our results lead us to suggest a hypothesis that would bridge the gap between the ice age theory and the phylogenetic results. We suggest that age-based emergence evolved in the Magicicada before the Pleistocene ice age, but they either remained protoperiodic or periodic with a much shorter cycle length than what the present-day Magicicada exhibit (and more in line with the cycle lengths observed in other contemporary periodic insects). Then this cycle length was elongated due to the adverse environmental conditions encountered during Pleistocene glacial periods, and the ratchet effect prevented the Magicicada from evolving back to shorter cycle lengths during interglacials.

We have deliberately used simple models for growth and environmental change, because they are helpful in elucidating the mechanisms of evolution. However, while we do not model stochastic variation in the mean environmental condition, we do not believe adding such noise to a reasonable degree would change any of our conclusions. More specifically, we do model growth as a stochastic process dependent on the environmental condition so that there is variation in the local environment that each individual experiences, and the environmental parameter is thus measuring the average environmental condition. We note that some stability in the mean environmental condition over a reasonable time interval likely is necessary for the evolution of age-based emergence, because invasion becomes possible when the resident population exhibits a somewhat consistent pattern of protoperiodical emergence. The environmental cycles that we consider here occur over timescales of tens of thousands to a hundred thousand years. Our examples are consistent with the type of environmental scenarios likely encountered during the Pleistocene ice age, where large temperature changes occurred back and forth over long timescales as glacial periods and interglacials succeeded one another (Bintanja et al. 2005; Elderfield et al. 2012). In particular, the dominant en- vironmental cycles during the Pleistocene were 41,000 and 100,000 years (Bintanja and van de Wal 2008).

It is important to note that one of the key reasons why the Magicicada are locked into their long, synchronous life cycles is their almost complete lack of predator avoidance, which has been described as foolhardy behavior (Lloyd and Dybas 1966b). Because of this, individuals emerging on a different year to the main emergence of the cohort are heavily selected against. Most other contemporary (annual) cicadas are much more difficult to catch (Lloyd and Dybas 1966b) so that emergence in low numbers is less of a risk, and this might be one reason why periodicity is not a common feature for other insects.

There is evidence that the Magicicada have undergone occasional life-cycle accelerations, where a significant proportion of a population with a 17-year life cycle emerges 4 years ahead of schedule, and this switch in cycle length may be permanent (Kritsky 1987; Martin and Simon 1988; Simon et al. 2000). It has been suggested that this plasticity in the life-cycle length evolved as a response to overcrowding (White and Lloyd 1979; Lloyd et al. 1983). Recently, Blackwood et al. (2018) studied how competition and life-cycle switches affect brood formation. In this article, we have neither considered nor modeled how this plasticity in life-cycle length would have evolved. Rather, we focus on the origin of the long and synchronous life cycles. We stress that it would be exceedingly helpful for future studies to have a better understanding of the genetic mechanisms determining emergence in cicadas.

\section{Acknowledgments}

This research was funded by the Academy of Finland (grant 283486). We acknowledge grants of computer capacity from the Finnish Grid and Cloud Infrastructure (persistent identifier urn:nbn:fi:research-infras-2016072533).

\section{Literature Cited}

Alexander, R. D., and T. E. Moore. 1962. The evolutionary relationships of 17-year and 13-year cicadas, and three new species (Homoptera, Cicadidae, Magicicada). Miscellaneous Publication no. 121, Museum of Zoology, University of Michigan, Ann Arbor.

Bintanja, R., and R. S. W. van de Wal. 2008. North American ice-sheet dynamics and the onset of 100,000-year glacial cycles. Nature 454:869-872.

Bintanja, R., R. S. W. van de Wal, and J. Oerlemans. 2005. Modelled atmospheric temperatures and global sea levels over the past million years. Nature 437:125-128.

Blackwood, J. C., J. Machta, A. D. Meyer, A. E. Noble, A. Hastings, and A. M. Liebhold. 2018. Competition and stragglers as mediators of developmental synchrony in periodical cicadas. American Naturalist 192:479-489. 
Bulmer, M. G. 1977. Periodical insects. American Naturalist 111: 1099-1117.

Cox, R. T., and C. E. Carlton. 1988. Paleoclimatic influences in the evolution of periodical cicadas (Insecta: Homoptera: Cicadidae: Magicicada spp.). American Midland Naturalist 120:183-193.

-2003. A comment on gene introgression versus en masse cycle switching in the evolution of 13-year and 17-year life cycles in periodical cicadas. Evolution 57:428-432.

Dybas, H. S., and D. D. Davis. 1962. A population census of seventeenyear periodical cicadas (Homoptera: Cicadidae: Magicicada). Ecology 43:432-444.

Dybas, H. S., and M. Lloyd. 1962. Isolation by habitat in two synchronized species of periodical cicadas (Homoptera: Cicadidae: Magicicada). Ecology 43:444-459.

- 1974. The habitats of 17-year periodical cicadas (Homoptera: Cicadidae: Magicicada spp.). Ecological Monographs 44:279324.

Elderfield, H., P. Ferretti, M. Greaves, S. Crowhurst, I. N. McCave, D. Hodell, and A. M. Piotrowski. 2012. Evolution of ocean temperature and ice volume through the mid-Pleistocene climate transition. Science 337:704-709.

Fortelius, M., S. Geritz, M. Gyllenberg, and J. Toivonen. 2015. Adaptive dynamics on an environmental gradient that changes over a geological time-scale. Journal of Theoretical Biology 376:91-104.

Grönholm, T., and A. Annila. 2007. Natural distribution. Mathematical Biosciences 210:659-667.

Heliövaara, K., R. Väisänen, and C. Simon. 1994. Evolutionary ecology of periodical insects. Trends in Ecology and Evolution 9:475-480.

Holling, C. S. 1959. Some characteristics of simple types of predation and parasitism. Canadian Entomologist 91:385-398.

Hoppensteadt, F. C., and J. B. Keller. 1976. Synchronization of periodical cicada emergences. Science 194:335-337.

Ito, H., S. Kakishima, T. Uehara, S. Morita, T. Koyama, T. Sota, J. R. Cooley, and J. Yoshimura. 2015. Evolution of periodicity in periodical cicadas. Scientific Reports 5:14094, doi:10.1038/srep14094.

Ito, Y., and M. Nagamine. 1981. Why a cicada, Mogannia minuta Matsumura, became a pest of sugarcane: an hypothesis based on the theory of "escape." Ecological Entomology 6:273-283.

Karban, R. 1982. Increased reproductive success at high densities and predator satiation for periodical cicadas. Ecology 63:321-328.

. 1984. Opposite density effects of nymphal and adult mortality for periodical cicadas. Ecology 65:1656-1661.

Kisdi, E., and S. A. H. Geritz. 1999. Adaptive dynamics in allele space: evolution of genetic polymorphism by small mutations in a heterogeneous environment. Evolution 53:993-1008.

Koch, A. L. 1966. The logarithm in biology. 1. Mechanisms generating the log-normal distribution exactly. Iournal of Theoretical Biology 12:276-290.

Kritsky, G. 1987. An historical analysis of periodical cicadas in Indiana (Homoptera:Cicadidae). Proceedings of the Indiana Academy of Science 97:295-322.

Levinton, J. S. 2001. Genetics, paleontology, and macroevolution. 2nd ed. Cambridge University Press, Cambridge.

Lloyd, M., and H. S. Dybas. 1966a. The periodical cicada problem. I. Population ecology. Evolution 20:133-149.
. 1966b. The periodical cicada problem. II. Evolution. Evolution 20:466-505.

Lloyd, M., G. Kritsky, and C. Simon. 1983. A simple Mendelian model for 13- and 17-year life cycles of periodical cicadas, with historical evidence of hybridization between them. Evolution 37:1162-1180.

Marshall, D. C., and J. R. Cooley. 2000. Reproductive character displacement and speciation in periodical cicadas, with description of a new species, 13-year Magicicada neotredecim. Evolution 54:1313-1325.

Martin, A. P., and C. Simon. 1988. Anomalous distribution of nuclear and mitochondrial DNA markers in periodical cicadas. Nature 336:237239.

- 1990. Temporal variation in insect life cycles: lessons from periodical cicadas. BioScience 40:359-367.

Osmond, M. M., and C. A. Klausmeier. 2017. An evolutionary tipping point in a changing environment. Evolution 71:2930-2941.

Ritterskamp, D., D. Bearup, and B. Blasius. 2016. Emergence of evolutionary cycles in size-structured food webs. Iournal of Theoretical Biology 408:187-197.

Ronce, O., and M. Kirkpatrick. 2001. When sources become sinks: migrational meltdown in heterogeneous habitats. Evolution 55:15201531.

Simon, C., J. Tang, S. Dalwadi, G. Staley, J. Deniega, and T. R. Unnasch. 2000. Genetic evidence for assortative mating between 13-year cicadas and sympatric "17-year cicadas with 13-year life cycles" provides support for allochronic speciation. Evolution 54:1326-1336.

Smith, B. E., and F. J. Merceret. 2000. The lognormal distribution. College Mathematics Journal 31:259-261.

Sota, T., S. Yamamoto, J. R. Cooley, K. B. R. Hill, C. Simon, and J. Yoshimura. 2013. Independent divergence of 13- and 17-y life cycles among three periodical cicada lineages. Proceedings of the National Academv of Sciences of the USA 110:6919-6924.

Toivonen, J., and L. Fromhage. 2018. Modeling the evolution of periodicity in the periodical cicadas. Evolutionary Ecology Research 19:107-125.

Valkenburgh, B. V. 2007. Déjà vu: the evolution of feeding morphologies in the Carnivora. Integrative and Comparative Biology 47:147-163.

Wahl, L. M. 2002. Evolving the division of labour: generalists, specialists and task allocation. Lournal of Theoretical Biology 219:371-388.

White, J. A., and M. Lloyd. 1975. Growth rates of 17 and 13-year periodical cicadas. American Midland Naturalist 94:127-143.

. 1979. 17-year cicadas emerging after 18 years: a new brood? Evolution 33:1193-1199.

White, J. A., M. Lloyd, and J. H. Zar. 1979. Faulty eclosion in crowded suburban periodical cicadas: populations out of control. Ecology 60:305-315.

Williams, K. S., and C. Simon. 1995. The ecology, behavior, and evolution of periodical cicadas. Annual Review of Entomology 40:269-295.

Yoshimura, J. 1997. The evolutionary origins of periodical cicadas during Ice Ages. American Naturalist 149:112-124.

Associate Editor: Jeremy W. Fox Editor: Russell Bonduriansky 